\title{
Dinámica y caracterización epidemiológica del brote de dengue en Argentina año 2016: el caso de la Provincia de Buenos Aires
}

\author{
Andrés Bolzan', Iván Insua², Carolina Pamparana³ ${ }^{3}$ María Celeste Giner 4 , Ana Medina ${ }^{5}$ y Betina Zucchino 6
}

\footnotetext{
'Dirección Provincial de

Epidemiología e Información Sistematizada, Ministerio de Salud de la Provincia de Buenos Aires, Argentina. ${ }^{2}$ Escuela Superior de Sanidad.

Dirección Provincial de Epidemiología y de Información Sistematizada.

32Dirección de Epidemiología, Ministerio de Salud de la Provincia

de Buenos Aires. Argentina.

${ }^{4}$ Dirección de Epidemiología Ministerio de Salud Provincia de Buenos Aires. La Plata, Argentina. ${ }^{5}$ Área de Epidemiología. Hospital

Interzonal Especializado de Agudos y Crónicos San Juan de

Dios. La Plata, Argentina.

${ }^{6}$ Ministerio de Salud BA. Subsecretaria de Contralor.
} Argentina.

El presente trabajo no registra conflicto de intereses. No tuvo aportes específicos para

la realización del estudio.

Recibido: 20 de junio de 2018 Aceptado: 2 de diciembre de 2018

Correspondencia a: Andrés Bolzan andresguillermobolzan@gmail.com

\section{Introducción}

$\mathrm{L}$ a fiebre del dengue es la principal enfermedad tropical emergente en el mundo, siendo la arbovirosis de mayor incidencia en el mundo, tanto en términos de morbilidad, como de mortalidad ${ }^{1}$. Según estimaciones de OMS, en las últimas décadas, la incidencia de dengue aumentó, aunque se sabe que el número real de casos de dengue está insuficientemente notificado y muchos casos están mal clasificados. Según una estimación reciente, se producen 390 millones de infecciones por dengue cada año (IC 95\%: 284 a 528 millones), de los cuales 96 millones (IC 95\%: 67 a 136 millones) se manifiestan clínicamente; esto significa que tres cuartas partes de la población permanece asintomáti$\mathrm{ca}^{2-6}$. Para el año 2016 se reportaron en las regiones de las Américas 1.679.537 casos de dengue (tasa de incidencia de 259,9 cada 100.000 habitantes) ${ }^{7}$, de los cuales más de 1.400.000 correspondieron al Cono Sur reportándose en total 577 muertes.

En Argentina, en las primeras 24 semanas epidemiológicas del año 2016, se notificaron 74.782 casos con sospecha de dengue de los cuales, 39.841 corres- pondieron a casos confirmados o probables autóctonos distribuidos en 15 provincias del país, mientras que 2.609 fueron casos confirmados y probables importados, distribuidos en 23 provincias $^{8}$. A comienzos de 1980 se produjo la reemergencia del dengue en el Cono Sur. Los primeros países vecinos de Argentina en notificar brotes de fiebre del dengue fueron Brasil en 1982 y $1986^{9}$, Bolivia en 1987-1988 y Paraguay en $1988-1989^{10}$. Chile experimentó brotes en Rapa Nui (antes Isla de Pascua), en 2002 y 2007, sin casos en el sector continental del país ${ }^{11}$. Otros países del Cono Sur registraron y reportaron la dinámica de la reinfestación ${ }^{12-16}$. La historia del dengue en Argentina se inicia en el año 1916, que afectó a 15.000 casos en la provincia de Entre Ríos, sin notificaciones de dengue hemorrágico ${ }^{17}$. En 1997 en la Provincia de Salta, y luego de más de 70 años sin notificaciones de dengue en el país ${ }^{18,19}$, se detectaron 19 casos en las localidades de Orán, Salvador Mazza, Güemes y Tartagal, producidos por el serotipo DEN-2. En años siguientes se reportaron varios brotes: en el año 1998 en la provincia de Salta con 359 casos confirmados por serotipo DEN-2 (se conocen actualmente 4 serotipos de diferente virulencia) en el 2000, en las provincias de 
Misiones y Formosa, con 617 casos, por serotipo DEN-1 -estando ambas epidemias vinculadas a brotes en países vecinos-20-22. Para el año 2002, nuevamente en Salta, se detectaron 214 casos por DEN-1, y en Misiones aparece el serotipo DEN-3 ${ }^{23}$. Hasta el año 2009, se continuaron reportando casos de DEN-1, 2 y 3 en las provincias de Formosa, Jujuy y Salta, Misiones y Corrientes. Pero hacia el año 2009, se produjo el mayor brote de dengue hasta el momento en la Argentina, con 26.923 casos confirmados de DEN-1 durante el primer semestre ${ }^{20}$, extendiéndose a 14 provincias de la Argentina, 10 de las cuales no contaban con historia epidemiológica previa de dengue ${ }^{21-23}$.

La recolonización de Aedes aegypti, en la Argentina siguió patrones similares a lo ocurrido en gran parte del continente y el resto del mundo ${ }^{24-28}$. En 1986, la reinfestación en Argentina se reportó en Posadas, Misiones, y luego en Clorinda y Puerto Pilcomayo en Formosa ${ }^{29}$. Para 1993 ya se encontraba en la Provincia de Buenos Aires $^{29}$ y en la Capital Federal en $1995^{30-34}$. Para el año 2000 se sumó la provincia de Mendoza ${ }^{31}$ y luego en 2006 hacia el sur la provincia de La Pampa incluyó la presencia del vector ${ }^{32}$. Recientemente se ha reportado la presencia del vector en la Provincia de Buenos Aires más allá de la isoterma de $15^{\circ} \mathrm{C}$, la que era considerada como factor limitante ${ }^{35,36}$. Para el caso de la Provincia de Buenos Aires, entre los posibles factores que facilitaron la re-colonización y proliferación vectorial se encuentran la urbanización explosiva ${ }^{29,32}$, las condiciones de mayor favorecimiento climático para el vector, el incremento en la producción o uso doméstico de productos descartables cuyos recipientes o envases pueden funcionar como criaderos y las deficiencias en la educación ambiental que permita prevenir la proliferación del vector ${ }^{24}$.

Hacia fines del año 2015 e inicios de 2016 se produce en la Argentina un brote de dengue que superó las cifras previamente notificadas para el país. En las primeras 15 semanas los casos acumulados superaron en $49 \%$ lo registrado en el brote del año 2009. El objetivo del presente trabajo busca caracterizar el brote de dengue ocurrido en la Provincia de Buenos Aires, de enero a mayo del año 2016 (semanas epidemiológicas 1 a 17).

\section{Material y Método}

\section{Marco geográfico}

La Provincia de Buenos Aires es una de las 24 provincias del territorio argentino y se encuentra en la región centro-este del país; limita al norte con la Provincia de Santa Fe y Entre Ríos; al noreste con el Río de la Plata y la Ciudad Autónoma de Buenos Aires. Con 15.625.000 habitantes, es la provincia más poblada de la Argentina (Figura 1).

\section{Fuente de información y confirmación de casos de dengue}

Fueron analizados todos los casos notificados al Sistema Nacional de Vigilancia de la Salud (SNVS) El SNVS constituye el sistema oficial en línea de denuncia y registro de las enfermedades infecto-contagiosas en el país. Incluye tanto la vigilancia de laboratorio como la de denuncia obligatoria. Las notificaciones quedaron agrupadas asignándolas como caso confirmado (laboratorio positivo) y caso no confirmado (laboratorio no positivo); dentro de este último grupo se incluyeron los casos sospechosos, los en estudio y los negativos, de forma de dicotomizar rápidamente la evolución del brote basándose en las confirmaciones efectivas por laboratorio.

\section{Análisis epidemiológico}

Se calcularon las tasas poblacionales respectivas de los tres agrupamientos: notificaciones compatibles con dengue, casos confirmados y casos no confirmados. Se calcularon los riesgos relativos y fracciones atribuibles respecto de los casos alóctonos (personas que viajaron a zonas endémicas y regresaron con síntomas a su zona de residencia en la Provincia de Buenos Aires) y autóctonos (personas que no viajaron a zonas endémicas y presentaron síntomas compatibles con dengue en su zona de residencia) para observar la evolución temporal de la incidencia acumulada. La definición de casos autóctonos y alóctonos se realizó a partir del registro epidemiológico en el SNVS, identificando el domicilio, si hubo viaje previo, el país donde se viajó y la fecha. La onda epidémica fue calculada partiendo de los datos de las fechas de inicio de síntomas de todos los casos notificados sospechosos y compatibles con dengue que proveía el SNVS, el período

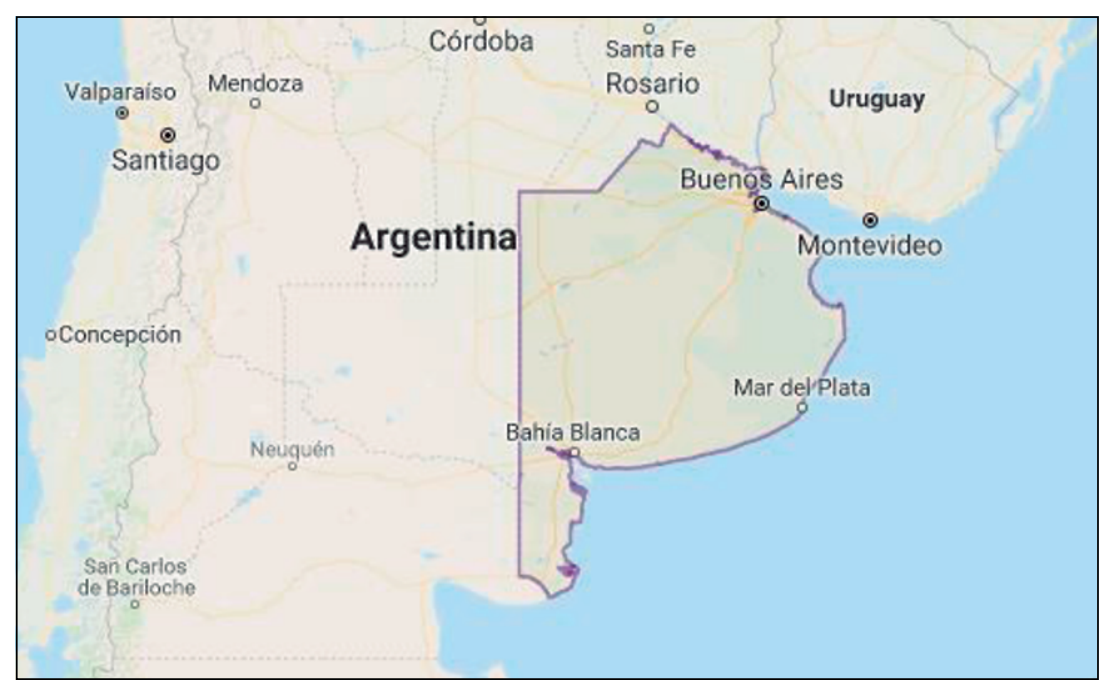

Figura 1. Mapa de ubicación de la Provincia de Buenos Aires, Argentina y sus límites. 
potencial de transmisión ( dose (curtosis, asimetría y coeficiente de variación) y de la onda (tasa de propagación y coeficiente de velocidad). Asimismo, con el objeto de considerar e identificar las zonas geográficas de mayor incidencia de casos confirmados, se calculó la distribución porcentual de tasas y casos acumulada mediante un gráfico distribucional acumulado de Pareto por Municipio de residencia.

\section{Geo-referenciamiento y análisis espacial}

Se trabajó tratando las notificaciones de dengue como un fenómeno puntual, ya que se ubicaron los casos confirmados y probables en un mapa del conurbano bonaerense según las direcciones de cada uno. Se geo-referenciaron manualmente por domicilio todos los casos ubicables, con una precisión de $50 \mathrm{~m}$, en Arcgis, con cartografia de base BingMaps y Open Street Map. La distribución de los casos por semana epidemiológica, podría reflejar un patrón de distribución homogéneo, o bien, un patrón de distribución heterogéneo en el espacio, lo que es igual a decir que existiría agregación de la variable analizada. Para ello, se analizó cómo se distribuyó la variable en el espacio mediante el algoritmo de vecino más cercano; este método verifica si los eventos epidemiológicos, exhiben conglomerados, es decir, una dispersión estadísticamente significativa en un rango de distancias. Luego, para medir la concentración o intensidad de agrupamientos de casos, se hizo un análisis de densidad Kernell por semana epidemiológica en el área del conurbano bonaerense con un tamaño de pixel de $100 \mathrm{~m}$ y un radio de búsqueda de $2.000 \mathrm{~m}$. Las superficies de densidad muestran dónde se concentran las entidades de punto. Se estableció en $100 \mathrm{~m}$ el tamaño pixel, dada la precisión de $50 \mathrm{~m}$ en la geo-codificación y que la distancia de vuelo o dispersión del mosquito es de $200 \mathrm{~m}$. Y el radio de búsqueda en $2.000 \mathrm{~m}$, dado que, del análisis de vecino más cercano realizado, la distancia media observada fue de $1.092 \mathrm{~m}$ y la distancia media esperada de $1.884 \mathrm{~m}$, el que fue redondeado a $2.000 \mathrm{~m}$.

Para los cálculos de vecino más próximo y densidad, se proyectaron los casos al sistema de referencia cartográficas planas Posgar 98 faja 5. Estos datos se emplearon para el análsis de conglomerados mediante método de Knox. Los intervalos usados fueron cortes naturales en cada semana, según la distribución de los casos (haciendo una reclasificación cualitativa: bajo, intermedio, moderado y alto), sin tener en cuenta los límites numéricos, dado que la cantidad de casos era distinta para cada semana.

\section{Caracterización espacio-temporal}

Se empleó el método de Knox para la detección de agregaciones espacio-temporales de los casos notificados y como subgrupo los casos confirmados de dengue del período diciembre de 2015 a fines de marzo de 2016. El método contrasta la hipótesis nula de que no existen pares de casos con agregación espacio-temporal. Para aplicar el método de Knox, se incluyeron todos los casos con datos geográficos de domicilio, resultado de laboratorio y fecha de inicio de síntomas. Se constituyeron en pares de coordenadas espaciales X e Y correspondientes a cada uno de los casos notificados ocurridos durante el período de estudio. Estas coordenadas se determinan en un sistema cartesiano. Para calcular la distancia espacial entre dos casos $\left(\mathrm{x}_{\mathrm{i}}, \mathrm{y}_{\mathrm{i}}\right)$ y $\left(\mathrm{x}_{\mathrm{j}}, \mathrm{y}_{\mathrm{j}}\right)$ se utiliza la distancia euclídea. Se fijaron como valores críticos $e=200$ metros y $t=5$ días, los cuales constituyen, respectivamente, la distancia máxima aceptada entre dos casos para que puedan ser considerados cercanos espacialmente (distancia espacial crítica) y el tiempo máximo entre la ocurrencia de dos casos para considerarse próximos en tiempo (distancia temporal crítica). Se realizó un análisis de sensibilidad para diferentes puntos de corte de $e$ y $t$. El procedimiento consiste en calcular las distancias espaciales y temporales entre todos los pares de casos y, a partir de los valores críticos establecidos, definir dos variables dicotómicas que expresan si un par de casos están o no próximos en el espacio y en el tiempo. La distancia temporal se estimó como la diferencia, en valor absoluto, entre las fechas de ocurrencia.

\section{Resultados}

Se registraron 4.844 notificaciones de dengue en la Provincia de Buenos Aires durante las primeras 17 semanas del año 2016, de los cuales 1.370 fueron casos confirmados por laboratorio (Tabla 1). En las primeras cinco semanas fueron notificados 709 casos, de los cuales 100 fueron confirmados por laboratorio, con una tasa de casos confirmados por cien mil habitantes de 0,64 que llegó a 8,79 hacia la semana 17, en tanto la tasa de casos notificados, de 4,55 a 31,07. La razón de tasas entre casos confirmados y notificados fue descendiendo a lo largo del tiempo, de 6,1 a 2,5 casos notificados por cada caso confirmado.

En el mismo sentido, la razón de tasas entre los casos confirmados alóctonos -cuyo contagio fue proveniente de zonas de viaje hacia la zona de residencia- y la de los casos autóctonos -contagio in situ en la zona de residencia- fue decreciendo de 8,8 a 0,4 (Tabla 2). El riesgo elevado de contagio por viaje a zonas endémicas se expresa mediante la fracción atribuible en los expuestos (viajeros), que claramente pasó de 0,91 en los inicios a 0,27 en la semana 17 (Tabla 3). En términos absolutos, mientras que las primeras cinco semanas del año se confirmaron 106 casos alóctonos y 12 autóctonos, hacia la semana 17 se invirtió la relación pasando a ser casi tres veces superior el contagio en la zona de residencia (938 casos autóctonos y 370 alóctonos, Tabla 3). 


\begin{tabular}{|c|c|c|c|c|c|c|c|c|c|c|c|}
\hline \multirow[b]{2}{*}{ RS } & \multicolumn{3}{|c|}{$\mathrm{CN}(\mathrm{n})$} & \multirow[t]{2}{*}{ Población } & \multicolumn{4}{|c|}{ Tasas } & \multicolumn{3}{|c|}{$C C(n)$} \\
\hline & $\mathrm{CC}$ & NC & Total & & Tasa C & Tasa N & Tasa NC & $\begin{array}{l}\text { Razón de } \\
\text { tasas NC/C }\end{array}$ & $\begin{array}{l}\text { Razón } \\
\mathrm{Al} / \mathrm{Au}\end{array}$ & $\mathrm{Au}$ & Al \\
\hline 1 & 2 & 24 & 26 & 655.133 & 0,31 & 3,97 & 3,66 & 11,81 & - & 0 & 2 \\
\hline$\|$ & 2 & 30 & 32 & 261.290 & 0,77 & 12,25 & 11,48 & 14,91 & 0,00 & 2 & 0 \\
\hline IV & 10 & 56 & 66 & 561.270 & 1,78 & 11,76 & 9,98 & 5,61 & 4,00 & 2 & 8 \\
\hline IX & 1 & 19 & 20 & 311.004 & 0,32 & 6,43 & 6,11 & 19,09 & 0,00 & 1 & 0 \\
\hline v & 166 & 470 & 636 & 3.139 .519 & 5,29 & 20,26 & 14,97 & 2,83 & 0,42 & 117 & 49 \\
\hline$x$ & 9 & 37 & 46 & 322.025 & 2,79 & 14,28 & 11,49 & 4,12 & - & 0 & 9 \\
\hline$X I$ & 119 & 272 & 391 & 1.172 .240 & 10,15 & 33,35 & 23,20 & 2,29 & 2,13 & 38 & 81 \\
\hline XII & 233 & 390 & 623 & 1.770 .240 & 13,16 & 35,19 & 22,03 & 1,67 & 0,33 & 175 & 58 \\
\hline Total & 1.370 & 3.474 & 4.844 & 15.592 .538 & 8,79 & 31,07 & 22,28 & 2,53 & 0,38 & 996 & 374 \\
\hline
\end{tabular}

RS: Región Sanitaria; CN: casos notificados; CC: casos confirmados por laboratorio; NC: casos no confirmados por laboratorio; Población: población total en habitantes de la RS; Tasa C: tasa poblacional de casos confirmados por 100 mil habitantes; Tasa N: tasa poblacional de casos notificados por 100 mil habitantes; TNC: tasa poblacional de casos no confirmados por 100 mil habitantes; Au: casos autóctonos; Al: casos alóctonos.

Los valores de la curva epidémica mostraron un aceleramiento en la tasa de propagación y en el coeficiente de difusión las primeras cinco semanas y, paulatinamente, el proceso de la curva de infección varió su curtosis y asimetría reflejando un proceso de expansión de los casos en el tiempo, aplanándose la curva y cayendo finalmente a valores menores a los iniciales en términos de confirmación de casos hacia principios del mes de mayo (Tabla 4 y Figura 5) .

Los datos geo-referenciados en tiempo y espacio mostraron conglomerados claramente identificados en los mapas secuenciales, constatándose, tanto por el análisis de Knox como la densidad Kernell, que unas pocas jurisdicciones presentaban acumulación de casos confirmados (Figuras 2 a 4).

Considerando un plazo de cinco días como media temporal del período de incubación e iniciando en $200 \mathrm{~m}$ el radio de desplazamiento del vector, hubo 43 pares de casos cercanos en tiempo y espacio, lo que en el análisis de sensibilidad se prolongó hasta encontrar pares cercanos en tiempo máximo de 30 días y espacio en los $1.600 \mathrm{~m}$. Estos pares cercanos se concentraron en ocho jurisdicciones con $73 \%$ de los casos confirmados (Tabla 5).
Tabla 2. Evolución del brote de dengue según acumulados por semanas epidemiológicas. Enero-mayo de 2016. Provincia de Buenos Aires

\begin{tabular}{ccccccc}
\hline $\begin{array}{c}\text { Tiempo } \\
\text { (semanas) }\end{array}$ & CN & CC & $\begin{array}{c}\text { Tasa CC } \\
(* 100 \text { mil })\end{array}$ & $\begin{array}{c}\text { Tasa CN } \\
(* 100 ~ m i l)\end{array}$ & \multicolumn{2}{c}{ Razón de tasas } \\
$\mathbf{N C} / \mathbf{C}$ & Al/Au \\
S1 - S5 & 709 & 100 & 0,64 & 4,55 & 6,10 & - \\
\hline S1 - S6 & 951 & 119 & 0,76 & 6,1 & 7,03 & 8,81 \\
\hline S1 - S7 & 1.177 & 164 & 1,05 & 7,55 & 6,28 & 5,84 \\
\hline S1 - S8 & 1.763 & 200 & 1,28 & 11,31 & 7,81 & 4,12 \\
\hline S1 - S9 & 2.145 & 293 & 1,88 & 13,76 & 6,34 & 2,24 \\
S1 - S10 & 2.515 & 355 & 2,28 & 16,13 & 6,10 & 1,61 \\
\hline S1 - S11 & 3.044 & 513 & 3,29 & 19,52 & 4,90 & 0,84 \\
\hline S1 - S12 & 3.314 & 628 & 4,03 & 21,25 & 4,34 & 0,62 \\
\hline S1 - S15 & 4.651 & 1.058 & 6,79 & 29,83 & 3,47 & 0,57 \\
\hline S1 - S17 & 4.844 & 1.370 & 8,79 & 31,07 & 2,54 & 0,44 \\
\hline
\end{tabular}

S: semanas epidemiológicas; CN: casos notificados; CC: casos confirmados; NC/C: tasas casos no confirmados/casos confirmados; Al/Au: tasas casos alóctonos/casos autóctonos. 
Tabla 3. Distribución acumulada según semanas epidemiológicas de tasas de incidencia de dengue por característica de viaje previo a zonas endémicas antes del inicio de síntomas

\begin{tabular}{|c|c|c|c|c|c|c|c|c|}
\hline \multirow{3}{*}{$\begin{array}{l}\text { Tiempo } \\
\text { (semanas) } \\
\text { S1 - S5 }\end{array}$} & \multicolumn{2}{|c|}{ Tasa de incidencia } & & & \multirow{2}{*}{\multicolumn{2}{|c|}{$\mathrm{Fr}$}} & \multicolumn{2}{|c|}{$C C(n)$} \\
\hline & Iv & Inv & \multicolumn{2}{|c|}{ RR (IC 95\%) } & & & $V p$ & Nvp \\
\hline & 0,26 & 0,02 & 11,1 & $(6,2-19,9)$ & 0,91 & $(0,83-0,94)$ & 106 & 12 \\
\hline $\mathrm{S} 1-\mathrm{S} 6$ & 0,26 & 0,03 & 7,50 & $(4,9-11,4)$ & 0,86 & $(0,79-0,91)$ & 140 & 24 \\
\hline $\mathrm{S} 1-\mathrm{S} 7$ & 0,23 & 0,04 & 6,10 & $(4,3-8,5)$ & 0,83 & $(0,56-0,67)$ & 161 & 39 \\
\hline $\mathrm{S} 1-\mathrm{S} 8$ & 0,24 & 0,07 & 3,20 & $(2,5-4,0)$ & 0,68 & $(0,61-0,75)$ & 193 & 109 \\
\hline S1 - S9 & 0,24 & 0,09 & 2,60 & $(2,2-3,2)$ & 0,62 & $(0,54-0,68)$ & 205 & 163 \\
\hline $\mathrm{S} 1-\mathrm{S} 10$ & 0,23 & 0,13 & 1,81 & $(1,55-2,11)$ & 0,44 & $(0,35-0,52)$ & 234 & 286 \\
\hline S1 - S11 & 0,26 & 0,17 & 1,51 & $(1,31-1,74)$ & 0,33 & $(0,23-0,42)$ & 247 & 388 \\
\hline $\mathrm{S} 1-\mathrm{S} 12$ & 0,24 & 0,17 & 1,34 & $(1,18-1,53)$ & 0,25 & $(0,15-0,34)$ & 279 & 460 \\
\hline S1 - S15 & 0,26 & 0,21 & 1,27 & $(1,13-1,42)$ & 0,21 & $(0,12-0,29)$ & 345 & 708 \\
\hline S1 - S17 & 0,28 & 0,27 & 0,28 & $(0,90-1,20)$ & 0,27 & $(0,15-0,22)$ & 370 & 938 \\
\hline
\end{tabular}

Tabla 4. Evolución y caracterización de la onda epidémica a lo largo del tiempo según semanas epidemiológicas.

\begin{tabular}{|c|c|c|c|c|c|c|c|c|c|}
\hline \multirow[t]{2}{*}{ Tiempo } & \multirow[t]{2}{*}{ Ndet } & \multicolumn{5}{|c|}{ Curva epidemiológica } & \multicolumn{3}{|c|}{ Cf (IC 95\%) } \\
\hline & & Xti & Ds & Asimetría & Curtosis & CV & & & \\
\hline S1 - S5 & 40 & 26,7 & 7,6 & $-0,77$ & 3,06 & 28,7 & 2,20 & 2,10 & 2,30 \\
\hline S1 - S6 & 41 & 27,2 & 7,9 & $-0,70$ & 3,10 & 28,8 & 2,10 & 2,13 & 2,21 \\
\hline S1 - S7 & 48 & 29,3 & 8,7 & $-0,53$ & 2,96 & 29,6 & 2,00 & 1,90 & 2,20 \\
\hline $\mathrm{S} 1-\mathrm{S} 8$ & 55 & 34,8 & 10,4 & $-0,44$ & 2,69 & 29,8 & 1,65 & 1,63 & 1,67 \\
\hline S1 - S9 & 64 & 39,0 & 12,0 & $-0,21$ & 2,50 & 31,6 & 1,30 & 1,20 & 1,40 \\
\hline $\mathrm{S} 1-\mathrm{S} 10$ & 68 & 41,6 & 14,2 & $-0,14$ & 2,20 & 34,1 & 1,10 & 1,18 & 1,20 \\
\hline $\mathrm{S} 1-\mathrm{S} 11$ & 78 & 46,9 & 15,8 & $-0,12$ & 2,19 & 33,7 & 1,08 & 1,07 & 1,09 \\
\hline $\mathrm{S} 1-\mathrm{S} 12$ & 83 & 47,2 & 17,1 & $-0,05$ & 2,17 & 36,2 & 1,03 & 0,90 & 1,01 \\
\hline $\mathrm{S} 1-\mathrm{S} 15$ & 84 & 47,5 & 17,3 & $-0,06$ & 2,17 & 36,4 & 0,99 & 0,98 & 0,99 \\
\hline $\mathrm{S} 1-\mathrm{S} 17$ & 99 & 52 & 19,2 & $-0,14$ & 2,2 & 36,6 & 0,90 & 0,90 & 0,91 \\
\hline
\end{tabular}

Las tasas de incidencia y la frecuencia de casos muestran una disparidad relativa debido a diferencias de totales de población, incluso en zonas vecinas. La distribución acumulada muestra, por un lado, la mayor tasa de incidencia en los partidos de Lomas de Zamora,
Morón, Tres de Febrero y Hurlingham con 500 casos (36\% del total de casos confirmados). Sin embargo, la casuística agrega zonas de mayor circulación viral en estos partidos sumando La Matanza, La Plata, Lanus y General San Martín; exceptuando La Plata, partido situado hacia 


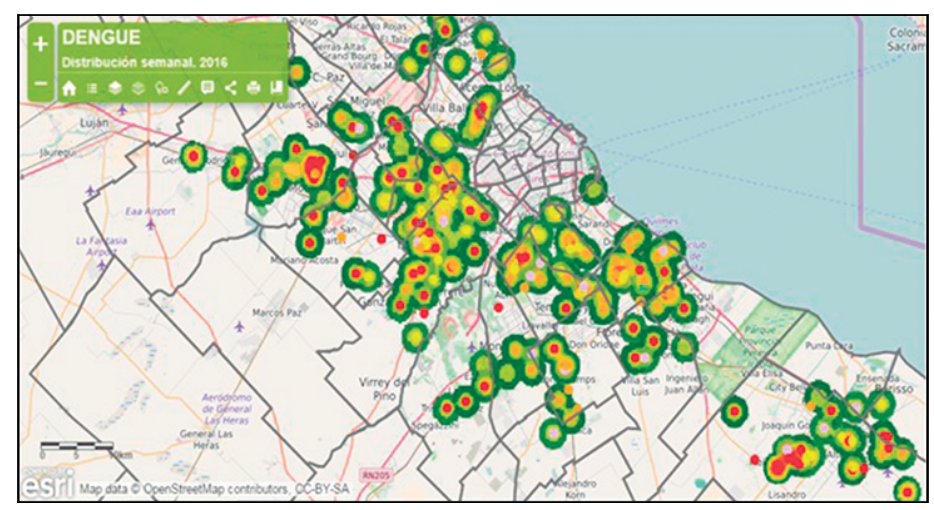

Figura 2. Distribución de agrupamientos temporo espaciales de casos de dengue mediante densidad Kernell. Primeras cuatro semanas epidemiológicas, año 2016. Buenos Aires, Argentina. Área del conurbano bonaerense (tamaño de pixel de 100 metros y un radio de búsqueda de 2.000 metros).

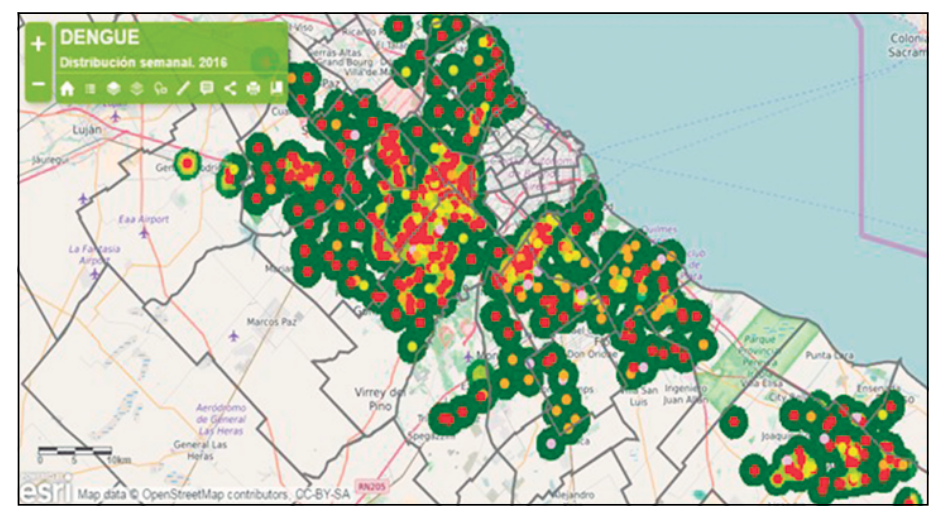

Figura 4. Distribución de agrupamientos temporo espaciales de casos de dengue mediante densidad Kernell. Semanas epidemiológicas novena a décimo segunda, año 2016. Buenos Aires, Argentina. Área del conurbano bonaerense (tamaño de pixel de 100 metros y un radio de búsqueda de 2.000 metros).

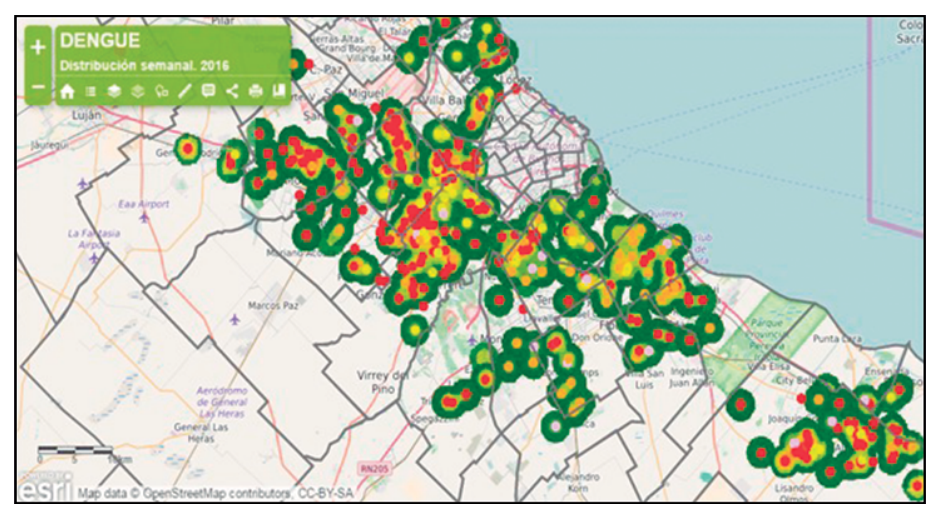

Figura 3. Distribución de agrupamientos temporo espaciales de casos de dengue mediante densidad Kernell. Semanas quinta a octava epidemiológicas, año 2016. Buenos Aires, Argentina. Área del conurbano bonaerense (tamaño de pixel de 100 metros y un radio de búsqueda de 2.000 metros).

el sur, este grupo conformó los conglomerados de mayor densidad. La circulación viral se concentró en los partidos del denominado Conurbano Bonaerense, las RS XII. VII y luego la XI y VI.

\section{Discusión}

El fenómeno de brote epidémico de dengue que experimentó la Argentina en el año 2016 no tuvo antecedentes comparables en la historia de la enfermedad en el país ${ }^{8,31,32,34,41,44}$. La Provincia de Buenos Aires mostró una dispersión de los casos confirmados por laboratorio centrados en conglomerados que constituyeron así las unidades de vigilancia focalizada e incluidas para posteriores acciones tendientes a disminuir la densidad vectorial en campañas de invierno. El seguimiento efectuado mediante el uso de esta metodología de epidemiología espacial permitió la intervención en los municipios. El conocimiento de la dispersión de

\begin{tabular}{|c|c|c|c|c|c|c|c|c|}
\hline Pares & $\begin{array}{c}e=200 \\
t=5\end{array}$ & $\begin{array}{c}e=200 \\
t=10\end{array}$ & $\begin{array}{c}e=400 \\
t=10\end{array}$ & $\begin{array}{c}e=800 \\
t=15\end{array}$ & $\begin{array}{c}e=2.000 \\
t=5\end{array}$ & $\begin{array}{c}e=2.000 \\
t=10\end{array}$ & $\begin{array}{c}e=1.600 \\
t=30\end{array}$ & $\begin{array}{c}e=3.000 \\
t=60\end{array}$ \\
\hline $\mathrm{Ce}+\mathrm{t}$ & 43 & 49 & 87 & 258 & 208 & 699 & 886 & 2.619 \\
\hline $\mathrm{Ce}-\mathrm{t}$ & 32 & 26 & 72 & 140 & 964 & 773 & 220 & 2 \\
\hline$A e+t$ & 18778 & 33.429 & 33.391 & 45.347 & 18.313 & 32.779 & 70.017 & 95.050 \\
\hline$A e-t$ & 79050 & 64.399 & 64.353 & 52.158 & 78.118 & 63.652 & 26.780 & 232 \\
\hline Total pares $(n *(n-1 / 2)$ & 97903 & & & & & & & \\
\hline Pares esperados $\mathrm{Ce}+\mathrm{t}$ & 14 & 25 & 54 & 185 & 282 & 503 & 801 & 2.614 \\
\hline Valor $p$ & $<0,001$ & $<0,001$ & $<0,001$ & $<0,001$ & $<0,001$ & $<0,001$ & 0,002 & 0,46 \\
\hline
\end{tabular}




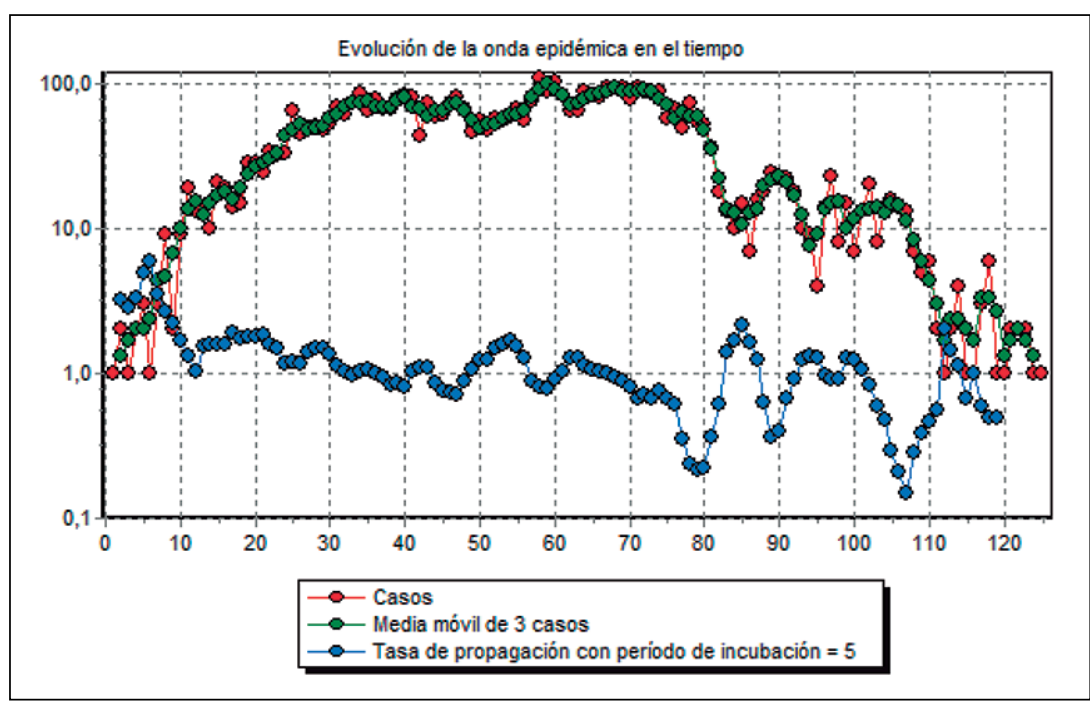

Figura 5. Evolución de la Onda epidémica de dengue, enero-mayo de 2016, Provincia de Buenos Aires. Casos notificados según semana de inicio de síntomas.

las personas en los barrios y del vector durante el brote es fundamental para comprender la dinámica de la enfermedad y su transmisión entre la población ${ }^{44,45}$. El empleo de la herramienta ArcGis, el análisis de Knox y el seguimiento de las densidades Kernell fueron dos herramientas de epidemiología espacial utilizadas en muchos estudios de áreas endémicas, de reciente recomendación en modelos predictores en Argentina y de prometedora evolución para la vigilancia epidemiológica del dengue y otras enfermedades vectoriales ${ }^{46-48}$. Sin embargo, no se contó con datos de densidad vectorial recientes para las áreas identificadas como de mayor circulación de la infección, por lo que algunos modelos de regresión no pudieron ser empleados. No obstante, el valor de estos índices ha sido discutido, si bien los rangos para el índice de vivienda de 4 a 35 e índice de Breteau de 5 a 50 han sido asociados con el riesgo de brotes epidémicos de dengue, dichos índices son un indicativo deficiente de la producción de adultos y de uso limitado a la hora de evaluar la abundancia del vector y el riesgo de transmisión de dengue ${ }^{21,49,50}$. Aun bajo esta consideración, los índices son empleados junto a otros factores ecosistémicos que amplían la información disponible y que, en cierta medida, explicaría el origen de estos índices. La evidencia de muchas investigaciones a nivel mundial, relacionan tres variables principales a la alta incidencia histórica de dengue y a valores elevados de infestación local de $A$. aegypti: la presión de vapor (una variable que combina la humedad y la temperatura), la densidad poblacional humana, y la disponibilidad en el medio ambiente de recipientes que acumulen agua ${ }^{49,51,52}$. A los fines del control vectorial, la magnitud de la actividad de dispersión de $A$. aegypti aún no está claramente definida, en tanto se reportan distancias de entre 100-800 metros - un rango de mucha variación-en Brasil ${ }^{16}$, mientras otros autores muestran datos entre 10 y 500 metros $^{53,54}$. En el presente estudio hemos considerado 200 metros como valor inicial para evaluar la distancia espacial entre los casos confirmados junto al período de incubación de cinco días, aunque aun elevando dicha distancia en metros hasta poco más de los 800 metros identificados en Río de Janeiro, los conglomerados persistieron como estadísticamente asociados. El análisis espacial -auto-correlación, análisis de conglomerado, serie temporal-son algunos de los métodos más empleados para testear si hay un patrón de dispersión en áreas particulares ${ }^{38}$. El empleo de las herramientas de SIG en la detección de conglomerados y áreas de densidad diferenciales son relevantes para detectar las denominadas "zonas calientes" de vigilancia epidemiológica activa. A partir de los hallazgos en la Provincia, se pudieron identificar estas zonas en el centro del conurbano bonaerense, particularmente en las llamadas Zonas Sanitarias VI, VII, XI y XII, donde se proyectó una campaña de invierno para el control del hábitat.

Un aspecto elemental y muy discutido es la densidad poblacional y la posibilidad de que el vector encuentre en estas aglomeraciones humanas un hábitat con alimento disponible y gran número de sitios de cría para sus estados inmaduros ${ }^{44}$. Aún más, se han relacionado ambos factores a la diferenciación genética de $A$. aegypti. El nivel de diferenciación genética entre las poblaciones de las afueras de la ciudad, donde la densidad humana es más baja y el agua se almacena en recipientes abiertos, es dos veces mayor que entre la población en las áreas del centro, donde la densidad humana es alta y hay menor permanencia de agua $^{53}$. En la ciudad de Buenos Aires, los sitios de mayor densidad urbana presentaron menor actividad de ovoposición que en aquellas zonas con mayor presencia de casas que departamentos ${ }^{54}$ ya que en la zona de departamentos suele haber menor disponibilidad de sitios de cría. En cambio, la relación positiva entre oviposición y sitios de industrias es relacionado con la disponibilidad de recipientes con agua permanente ${ }^{55}$, siendo, en cambio, los sitios menos expuestos a la luz solar (como podrían ser los microcentros urbanos) desfavorables. En parte, estos hechos muestran evidencia para poder seleccionar los sitios de campañas invernales focalizadas en la modificación parcial del hábitat del vector para hacerlo menos favorable, como la disponibilidad de agua en las viviendas con sectores de patio y pulmones de manzana. Las facilidades de circulación humana también constituyen parte del hábitat donde A. aegypti prospera. Por ejemplo, en Tartagal, provincia de Salta, el transporte generado por la cercana ruta 34 favoreció el crecimiento rápido del brote del año $2004^{44}$. Otro aspecto relacionado son los neumáticos descartados, que presentan depósitos de huevos del vector y que, además, son transportados 
mediante el tráfico terrestre hacia otras localidades ${ }^{44}$ expandiendo al vector y, por ende, las probabilidades de que nuevos susceptibles sean infectados determinan que los estudios epidemiológicos del dengue son complejos, en parte dado por la gran prevalencia de la transmisión silente, que según estimaciones podría estar cerca de $80 \%{ }^{54,55}$. Así, los casos reportados son los clínicamente evidentes y, por consiguiente, podríamos considerarlos los más graves. A este hecho se suma la efectividad de los sistemas de vigilancia, tanto de laboratorio como clínicos. Tampoco se conoce el estado inmunológico de la población humana respecto de la infección. Cada serotipo de dengue provee de inmunidad serotipo-específica en los casos que se recuperan, que son la gran mayoría de los pacientes, por lo que este aspecto es relevante.

Por otra parte, tampoco se conoce la capacidad vectorial de las poblaciones del vector en el área estudiada. La habilidad de una población de mosquitos para transmitir un patógeno es determinada por una serie de variables fisiológicas y ecológicas. Entre las propiedades relevantes de una población de mosquito se incluyen: competencia vectorial, densidad del vector, proporción de picaduras sobre humanos, período de incubación extrínseco y longevidad del vector ${ }^{57}$. Estos vectores determinan los niveles de transmisión del virus dengue a los seres humanos por lo que inciden directamente en la difusión y ocurrencia de esta enfermedad a nivel mundial.

En definitiva, este estudio aporta datos sobre el fenómeno reemergente de la infección por arbovirus dengue (principalmente DEN-1 circulante) en la Provincia de Buenos Aires durante los primeros cuatro meses del año 2016, con cifras de incidencia que superaron lo históricamente conocido en la historia de la enfermedad en Argentina. El empleo de tecnología de epidemiología espacial permitió localizar y hacer el seguimiento diario y semanal de la infección y su dispersión temporal y en distancia y establecer "zonas calientes" para priorizar acciones. Es patente la necesidad de mejorar los sistemas de alerta y la consistencia de la información consolidada, eso es, del sistema nacional de vigilancia (SNVS) y sus componentes C2 (alerta clínica) y SIVILA (alerta de laboratorio). La arbovirosis ha evidenciado que el problema del control vectorial es la clave para poder disminuir las tasas de incidencia locales y, además, con- trolar los casos alóctonos, sobre todo los provenientes de provincias como Misiones y Formosa, que son endémicas para el dengue.

Agradecimientos: este trabajo no habría sido posible sin la colaboración activa y necesaria del Sistema de Información Geográfico del Ministerio de Salud de la Provincia de Buenos Aires, el Centro de Estudios Parasitológicos y de Vectores (CONICET, UNLP) y el Instituto de Limnologia Dr. RA. Ringuelet (CONICET, UNLP), así como el aporte sustancial de la Comisión Nacional de Actividades Espaciales en la discusión de aportes con sensores remotos.

\section{Resumen}

Introducción: El dengue, de amplia distribución mundial, ha resurgido en Argentina tras más de 70 años de ausencia, con un comportamiento endémico en las provincias norteñas del país; durante el año 2016 se experimentó una diseminación epidémica a la Provincia de Buenos Aires. Objetivos: Caracterizar el brote de fiebre de dengue ocurrido en la Provincia de Buenos Aires, Argentina, durante el período de inicio y expansión del mismo entre enero y mayo del año 2016. Métodos: El Sistema Nacional de Vigilancia de la Salud proveyó los datos semanales, utilizándose, además, ArcGis para la localización espacial. Se empleó test de Knox para establecer relaciones de coordenadas temporo-espaciales y se diseñó la curva epidemiológica calculando el coeficiente de difusión. Resultados: El riesgo relativo y razones de tasas para casos confirmados y la razón infección fue caracterizada por un aumento rápido en su propagación, con conglomerados definidos acompañado de la modificación sostenida entre las razones de tasas de casos infectados provenientes de zonas endémicas y aquellos autóctonos. Hacia la semana 17, el brote cae teniendo los valores más bajos de difusión. Discusión: La información evaluada mediante diferentes sistemas de información y análisis permitió identificar debilidades de los mismos, así como visualizar "zonas calientes" de circulación del virus para determinar acciones tendientes a concentrar los esfuerzos de control de potenciales criaderos de estados inmaduros del mosquito en épocas invernales.

\section{Referencias bibliográficas}

1.- Organización Mundial de la Salud 2016. Carga mundial de Dengue. http:/www.who.int/ mediacentre/factsheets/fs117/es/ (accedido en octubre de 2018).

2.- Bhatt S, Messina J P, Brownstein J S, Hoen AG, et al. Refining the global spatial limits of Dengue virus transmission by evidence-based consensus. PLoS Negl Trop Dis 2012; 6: e1760. DOI: 10.1371/journal.pntd.0001760.

3.- Duonga V, Lambrechtsb L, Paul R E, Lye S, Laya R S, Kanya C. et al Asymptomatic humans transmit Dengue virus to mosquitoes. 2015. www.pnas.org/cgi/doi/10.1073/ pnas. 1508114112 .

4.- Bhatt $\mathrm{S}$. The global distribution and burden of Dengue. Nature 2013; 496 (7446): 504-7.
DOI: $10.1038 /$ nature 12060 .

5.- World Health Organization, 2010. DengueNet database and geographic information system. Geneva. http: // www.who.int/DengueNet (accedido en mayo de 2017).

6.- World Health Organization, 2010. Pandemic Influenza (H1N1) 2009. http://www.who.int/ csr/disease/swineflu/en (accedido en octubre de 2018). 
7.- PAHO. Number of reported cases of dengue and severe dengue (SD) in the Americas, by country: Epidemiological Week / EW 22 (Updated June 17, 2016).

8.- Argentina, Ministerio de Salud. Boletín Integrado de Vigilancia $N^{\circ} 314$, Buenos Aires 2016. https://www.argentina.gob.ar/sites/ default/files/boletin-integrado-de-vigilancian315-se25.pdf

9.- Schatzmayr H G. Dengue situation in Brazil by year 2000. Mem Inst Oswaldo Cruz 2000; 95: 179-81. http://dx.doi.org/10.1590/S007402762000000700030

10.- PAHO. Dengue and Dengue Hemorrhagic Fever in the Americas: Guidelines for Prevention and Control, PAHO Scientific Publication n. 548, Washington, 98 pp 1994.

11.- Ministerio de Salud de Chile 2007. Situación epidemiológica Dengue en Isla de Pascua, Chile insular, 18/04/2007. Departamento de Epidemiología, Ministerio de Salud, Chile. http://epi.minsal.cl/epi/html/bolets/reportes/ Dengue/InfSituacDENGUEchile.pdf.

12.- Correa P R, Franca E, Bogutchi T F. Aedes aegypti infestation and occurrence of Dengue in the city of Belo Horizonte, Brazil. Rev Saude Publica 2005; 39: 33-40. http://www.ncbi.nlm. nih.gov/pubmed/15654458.

13.- Fernández R W, Lannacone O J, Rodríguez P E, Salazar C N, Valderrama. Population behavior of Aedes aegypti larvae for estimating Dengue fever cases in Yurimaguas, Peru, 2000-2004. Rev Peru Med Exp Salud Publica 2005; 22 : 175-82. http://www.scielo.org.pe/pdf/rins/ v22n3/a04v22n3.pdf.

14.- Arboleda SS, Jaramillo-O NN, Peterson ATA. Spatial and temporal dynamics of Aedes aegypti larval sites in Bello, Colombia. J Vector Ecol 2012; 37: 37-48. DOI: 10.1111/j.19487134.2012.00198.x

15.- Sánchez L, Vanlerberghe V, Alfonso L, Marquetti M D C, Guzmán M G, et al. Aedes aegypti larval indices and risk for Dengue epidemics. Emerg Infect Dis. 2006; 12: 800-6. http://wwwnc.cdc.gov/eid/ article/12/5/05-0866_article.htm.

16.- Honorio N A, Nogueira R M R, Codeco C T, Carvalho M S, Cruz O G, et al. Spatial evaluation and modeling of Dengue seroprevalence and vector density in Rio de Janeiro, Brazil. Plos Negl Trop Dis 2009; http:// dx.doi.org/10.1371/journal.pntd.0000545.

17.- Gaudino N M. El Dengue: Algunas consideraciones sobre la epidemia de Entre Ríos de 1916. Rev Sanidad Mil 1916; 15: 617-27.

18.- Seijo A, Cernigoi B, Deodato B. Dengue importado del Paraguay a Buenos Aires. Medicina (Buenos Aires) 2001; 61: 137-41. http://www.medicinabuenosaires.com/revistas/ vol61-01/2/v61_n2_p137_141.pdf.

19.- Curto S, Bofia R, Carbajo A, Plastina R, Schweigmann N. Reinfestación del territorio argentino por Aedes aegypti. Distribución geográfica (1994-1999). Actualizaciones en Artropodología Sanitaria Argentina. Fundación Mundo Sano. ISBN 987-20421-0-1. Noviembre 2002.

20.- OPS. Number of reported cases of Dengue and Dengue hemorrhagic fever in the Americas, by country. Epidemiological Week / EW 52 (Updated 21 January 2011).

21.- Rotela C. Desarrollo de modelos e indicadores remotos de riesgo epidemiológico de Dengue en Argentina. Tesis doctoral, Instituto de Altos Estudios Espaciales "Mario Gulich" Comisión Nacional de Actividades Espaciales Universidad Nacional de Córdoba. Córdoba, Argentina 2012.

22.- Avilés G, Paz M V, Rangeon G, Ranaivoarisoa $\mathrm{M}$, Verzeri N, Roginski S, et al. Laboratory surveillance of Dengue in Argentina, 19952001. Emerg Infect Dis 2003: 9 (6): 738-42. doi: 10.3201/eid0906.020483.

23.- Argentina. Ministerio de Salud de la Nación. Boletín Epidemiológico Nacional, Dirección de Epidemiología, 2002.

24.- Schweigmann N, Rizzotti A, Castiglia G, Gribaudo F, Marcos M, Burroni N, et al Información, conocimiento y percepción sobre el riesgo de contraer el Dengue en Argentina: dos experiencias de intervención para generar estrategias locales de control. Cad. Saúde Pública. Rio de Janeiro 2009; 25 (Sup 1):13748. http://server.ege.fcen.uba.ar/gem/pdf/ Schweigmann\%20et\%20al.\%202009.pdf.

25.- Bejarano JFR. Estudio sobre la fiebre amarilla selvática en la República Argentina, Subsecretaria de Medicina Sanitaria, República Argentina 1979; 38 pp.

26.- Schatzmayr H G. Dengue situation in Brazil by year 2000. Mem Inst Oswaldo Cruz 2000; 95: 179-81. PMID: 11142711.

27.- Salvatella R, Rosa R. Culicidos y salud humana en el Uruguay. Entomol Vect 2003; 10: 431-6.

28.- Guedes D R, Cordeiro M T, Melo-Santos M A, Magalhaes T, Marques E, Regis L, et al. Patient-based dengue virus surveillance in Aedes aegypti from Recife. Brazil J Vector Borne Dis 2010; 47: 67-75. PMID: 20539043.

29.- Campos RE. Presencia de Aedes (Stegomyia) aegypti L. (Diptera: Culicidae) en la localidad de Quilmes (Buenos Aires, Argentina). Rev Soc Entomol Argent 1993; 52: 36. https://www.biotaxa.org/RSEA/article/ viewFile/32713/29055

30.- Vezzani D, Carbajo A E. Aedes aegypti, Aedes albopictus, and Dengue in Argentina: current knowledge and future directions. Mem Inst Oswaldo Cruz, Rio de Janeiro. 2008; 103(1): 66-74. PMID: 18327504

31.- Domínguez C, Lagos S. Presencia de Aedes aegypti (Diptera: Culicidae) en la provincia de Mendoza, Argentina. Rev Soc Entomol Argent 2001; 60: 79-80. https://www.biotaxa.org/
RSEA/article/viewFile/31995/28433

32.- Rossi G C, Lestani E A, D’Oria J M. Nuevos registros y distribución de mosquitos de la Argentina (Diptera: Culicidae). Rev Soc Entomol Argent 2006; 65: 51-56. http://www. scielo.org.ar/pdf/rsea/v65n3-4/v65n3-4a08.pdf

33.- Stein M, Oria G I, Almirón W R, Willener J A. Seasonal fluctuation of Aedes aegypti in Chaco Province, Argentina. Rev Saúde Pública 2005; 39: 559-64. http://dx.doi.org/10.1590/S003489102005000400007.

34.- Schweigmann N, Vezzani D, Orellano P, Kuruc J, Boffi R. Aedes albopictus in an area of Misiones, Argentina. Rev Saúde Pública 2004; 38: 136-8. PMID: 14963554

35.- Otero M, Solari H G, Schweigmann N. A stochastic population dynamics model for Aedes aegypti: and application to a city with temperate climate. Bull. Math. Biol 2006; 68: 1945-74. DOI: 10.1007/s11538-006-9067-y.

36.- Zanotti G, De Majo M S, Alem I, Schweigmann N, Campos R E, Fischer S. New records of Aedes aegypti at the southern limit of its distribution in Buenos Aires province, Argentina. J Vector Ecology 20015; 40 (2): 408-11. https://doi.org/10.1111/ jvec. 12181

37.- Ling Cheong Y, Burkart K, Leitão P J, Lakes T. Assessing weather effects on Dengue disease in Malaysia. Int. J. Environ. Res. Public Health 2013; 10: 6319-34. doi: 10.3390/ ijerph10126319.

38.- Phaisarn J, Nitin Kumar T, Souris M. Spatiotemporal diffusion pattern and hotspot detection of Dengue in Chachoengsao Province, Thailand Int. J. Environ. Res. Public Health 2011; 8: 5174. doi: $10.3390 /$ ijerph8010051.

39.- Cox J, Grillet M E, Ramos O M, Amador M, Barrera R, Habitat segregation of dengue vectors along an urban environmental gradient. Am J Trop Med Hyg 2007; 76(5): 820-26. PMID: 17488898.

40.- Silva F D, Dos Santos A M, Corrêa Rda G, Caldas A de J. Temporal relationship between rainfall, temperature and occurrence of Dengue cases in São Luís, Maranhão, Brazil. Cien Saude Colet 2016; 21 (2): 641-46. DOI: 10.1590/1413-81232015212.09592015.

41.- Bowman L R, Runge-Ranzinger S, McCall P $\mathrm{J}$. Assessing the relationship between vector indices and Dengue transmission: A systematic review of the evidence. PLoS Negl Trop Dis 2014; 8 (5): e2848.http://doi:10.1371/journal. pntd.0002848.

42.- Louis V R, Phalkey R, Horstick O, Ratanawong P, Wilder-Smith A, Tozan Y, et al. Modeling tools for dengue risk mapping - a systematic review Int J Health Geogr 2014; 13: 50. DOI: $10.1186 / 1476-072 X-13-50$.

43.- Rotela C, Fouque F, Lamfri M, Sabatier P, Introini V, Zaidenberg M, et al. Space-time analysis of the dengue outbreak spreading 
dynamics in the 2004 Tartagal outbreak, Northern Argentina. Acta Trop 2007; 103: 1-13. DOI: 10.1016/j.actatropica.2007.05.003.

44.- Harrington L C, Scott T W, Lerdthusnee K, Coleman R C, Costero A, Clark G G, et al. Dispersal of the dengue vector Aedes aegypti within and between rural communities. Am J Trop Med Hyg 2005: 72: 209-20. PMID: 15741559.

45.- Barmak D H, Dorso C O, Otero M, Solari H G. Dengue epidemics and human mobility. Phys Rev E Stat Nonlin Soft Matter Phys 2011; 84: (1 Pt 1): 011901. PMID: 21867207

46.- Nevai A L, Soewono E. A model for the spatial transmission of Dengue with daily movement between villages and a city. Math Med Biol 2014; 31 (2): 150-78. DOI: 10.1093/imammb/ dqt002.

47.- Epidemiología panorámica. Introducción al uso de herramientas geoespaciales aplicadas a la salud pública. Camilo Hugo Rotela. Comisión Nacional de Actividades Espaciales; Ministerio de Planificación Federal Inversión Pública y Servicios Ministerio de Salud de la Nación.
Dirección de Epidemiologia, Ciudad Autónoma de Buenos Aires 1 ${ }^{\mathrm{a}}$. Ed, 2014.

48.- Tauil P L. Urbanization and Dengue ecology. Cad Saude Publica 2001; 17 (Suppl): 99-102. doi:10.1590/S0102-311X2001000700018 PMID:11426270.

49.- Focks D A. A review of entomological sampling methods and indicators for Dengue vectors. Geneva, Switzerland: World Health Organization. whqlibdoc. who.int/hq/2003/ TDR_IDE_DEN_03.1.pdf. (accedido en octubre de 2018).

50.- Rubio-Palis Y, Pérez-Ybarra LM, InfanteRuiz M, Comach G, Urdaneta-Márquez L. Influence of climatic variables on Dengue cases and abundance of Aedes aegypti (Diptera: Culicidae) in Maracay, Venezuela. Bol Mal Salud Amb 2011; 51: 145-57.

51.- Pham H V, Doan H T M, Phan T T T, Minh N $\mathrm{N}$ T. Ecological factors associated with dengue fever in a central highlands province, Vietnam. Stoch. Environ Res Risk Assess 2011; 25: 48594. DOI: $10.1186 / 1471-2334-11-172$.

52.- Julio N B, Chiappero M B, Rossi H, Rondan
Dueñas J C, Gardenal C. Genetic structure of Aedes aegypti in the city of Córdoba (Argentina), a recently reinfested area. Mem Inst Oswaldo Cruz, Rio de Janeiro 2009; 104 (4): 626-31. http://www.bioline.org.br/ pdf?oc09141.

53.- Carbajo A E, Curto S I, Schweigmann N J. Spatial distribution pattern of oviposition in the mosquito Aedes aegypti in relation to urbanization in Buenos Aires: southern fringe bionomics of an introduced vector. Med Vet Entomol 2006; 20: 209-18. PMID: 16871702.

54.- Vezzani D, Rubio A, Velázquez S M, Schweigmann N, Wiegard T. Detailed assessment of microhabitat suitability for Aedes aegypti (Diptera: Culicidae) in Buenos Aires, Argentina. Acta Trop 2005; 95: 123-31. DOI: 10.1016/j.actatropica.2005.03.010.

55.- Chastel C. Infections inapparentes chez l'Homme: un cheval de Troie pour l'introduction et la diffusion des arbovirus transmis par des moustiques dans les régions non endémiques? Bull Soc Pathol Exot 2011; 104: 213-9. DOI: 10.1007/s13149-011-0165-1. 